\title{
Asymptotic Representation of the Solutions of Linear Volterra Difference Equations
}

\author{
István Győri and László Horváth \\ Department of Mathematics and Computing, University of Pannonia, 8200 Veszprém, \\ Egyetem u. 10, Hungary
}

Correspondence should be addressed to István Győri, gyori@almos.vein.hu

Received 26 February 2008; Accepted 4 April 2008

Recommended by Elena Braverman

This article analyses the asymptotic behaviour of solutions of linear Volterra difference equations. Some sufficient conditions are presented under which the solutions to a general linear equation converge to limits, which are given by a limit formula. This result is then used to obtain the exact asymptotic representation of the solutions of a class of convolution scalar difference equations, which have real characteristic roots. We give examples showing the accuracy of our results.

Copyright ( 12008 I. Győri and L. Horváth. This is an open access article distributed under the Creative Commons Attribution License, which permits unrestricted use, distribution, and reproduction in any medium, provided the original work is properly cited.

\section{Introduction}

The literature on the asymptotic theory of the solutions of Volterra difference equations is extensive, and application of this theory is rapidly increasing to various fields. For the basic theory of difference equations, we choose to refer to the books by Agarwal [1], Elaydi [2], and Kelley and Peterson [3]. Recent contribution to the asymptotic theory of difference equations is given in the papers by Kolmanovskii et al. [4], Medina [5], Medina and Gil [6], and Song and Baker [7]; see [8-19] for related results.

The results obtained in this paper are motivated by the results of two papers by Applelby et al. [20], and Philos and Purnaras [21].

This paper studies the asymptotic constancy of the solution of the system of nonconvolution Volterra difference equation

$$
z(n+1)=\sum_{i=0}^{n} H(n, i) z(i)+h(n), \quad n \in \mathbb{Z}^{+},
$$


with the initial condition

$$
z(0)=z_{0}
$$

where $z_{0} \in \mathbb{R}^{d},(H(n, i))_{0 \leq i \leq n}$ and $(h(n))_{n \geq 0}$ are sequences with elements in $\mathbb{R}^{d \times d}$ and $\mathbb{R}^{d}$, respectively.

Under appropriate assumptions, it is proved that the solution converges to a finite limit which obeys a limit formula. Our paper develops further the recent work [20]. The distinction between the works is explained as follows. For large enough $n$, in fact $n \geq 2 m+2$, the sum in (1.1) can be split into three terms

$$
\sum_{i=0}^{m} H(n, i) z(i)+\sum_{i=m+1}^{n-m-1} H(n, i) z(i)+\sum_{j=0}^{m} H(n, n-j) z(n-j),
$$

since

$$
\sum_{i=n-m}^{n} H(n, i) z(i)=\sum_{j=0}^{m} H(n, n-j) z(n-j) .
$$

In [20, Theorem 3.1] the middle sum in (1.3) contributed nothing to the $\operatorname{limit}_{\lim } \lim _{n \rightarrow \infty} z(n)$, since it was assumed that

$$
\lim _{m \rightarrow \infty}\left(\limsup _{n \rightarrow \infty} \sum_{i=m}^{n-m}|H(n, i)|\right)=O .
$$

In our case, we split the sum in (1.1) only into two terms, and the condition (1.5) is not assumed. In fact, we show an example in Section 4, where (1.5) does not hold and hence in [20, Theorem 3.1] is not applicable. At the same time our main theorem gives a limit formula. It is also interesting to note that our proof is simpler than it was applied in [20].

Once our main result for, the general equation, (1.1) has been proven, we may use it for the scalar convolution Volterra difference equation with infinite delay,

$$
\Delta x(n)=A x(n)+\sum_{j=-\infty}^{n} K(n-j) x(j)+g(n), \quad n \in \mathbb{Z}^{+},
$$

with the initial condition,

$$
x(n)=\varphi(n), \quad n \in \mathbb{Z}^{-},
$$

where $A \in \mathbb{R}$, and $(K(n))_{n \geq 0},(g(n))_{n \geq 0}$ and $(\varphi(n))_{n \leq 0}$ are real sequences.

Here, $\Delta$ denotes the forward difference operator to be defined as usual, that is, $\Delta x(n):=$ $x(n+1)-x(n), n \in \mathbb{Z}^{+}$.

If we look for a solution $x(n)=\lambda_{0}^{n}, n \in \mathbb{Z}^{+}\left(\lambda_{0} \in \mathbb{R} \backslash\{0\}\right)$ of the homogeneous equation associated with (1.6), we see that $\lambda_{0}$ is a root of the characteristic equation

$$
\lambda_{0}=1+A+\sum_{i=0}^{\infty} K(i) \lambda_{0}^{-i}
$$


We immediately observe that $\lambda_{0} \in \mathbb{R}$ is a simple root if

$$
\left|\lambda_{0}\right|^{-1} \sum_{i=1}^{\infty} i|K(i)|\left|\lambda_{0}\right|^{-i}<1
$$

In the paper [21] (see also [22]), it is shown that if $\lambda_{0}>0$ satisfies (1.8) and (1.9), and the initial sequence $(\varphi(n))_{n \leq 0}$ is suitable, then for the solution $x$ of (1.6) and (1.7) the sequence $z(n):=\lambda_{0}^{-n} x(n), n \in \mathbb{Z}^{+}$is bounded. Furthermore, some extra conditions guarantee that the limit $z(\infty):=\lim _{n \rightarrow \infty} z(n)$ is finite and satisfies a limit formula.

In our paper, we improve considerably the result in [21]. First, we give explicit necessary and sufficient conditions for the existence of a $\lambda_{0} \in \mathbb{R} \backslash\{0\}$ for which (1.8) and (1.9) are satisfied. Second, we prove the existence of the limit $z(\infty)$ and give a limit formula for $z(\infty)$ under the condition only $\lambda_{0} \neq 0$. These two statements are formulated in our second main theorem stated in Section 3. The proof of the existence of $z(\infty)$ is based on our first main result.

The article is organized as follows. In Section 2, we briefly explain some notation and definitions which are used to state and to prove our results. In Section 3, we state our two main results, whose proofs are relegated to Section 5.

Our theory is illustrated by examples in Section 4, including an interesting nonconvolution equation. This example shows the significance of the middle sum in (1.3), since only this term contributes to the limit of the solution of (1.1) in this case.

\section{Mathematical preliminaries}

In this section, we briefly explain some notation and well-known mathematical facts which are used in this paper.

Let $\mathbb{Z}$ be the set of integers, $\mathbb{Z}^{+}:=\{n \in \mathbb{Z} \mid n \geq 0\}$ and $\mathbb{Z}^{-}:=\{n \in \mathbb{Z} \mid n \leq 0\} . \mathbb{R}^{d}$ stands for the set of all $d$-dimensional column vectors with real components and $\mathbb{R}^{d \times d}$ is the space of all $d$ by $d$ real matrices. The zero matrix in $\mathbb{R}^{d \times d}$ is denoted by $O$, and the identity matrix by $I$. Let $E$ be the matrix in $\mathbb{R}^{d \times d}$ whose elements are all 1 . The absolute value of the vector $x=\left(x_{1}, \ldots, x_{d}\right)^{T} \in \mathbb{R}^{d}$ and the matrix $A=\left(A_{i j}\right)_{1 \leq i, j \leq d} \in \mathbb{R}^{d \times d}$ is defined by $|x|:=\left(\left|x_{1}\right|, \ldots,\left|x_{d}\right|\right)^{T}$ and $|A|:=\left(\left|A_{i j}\right|\right)_{1 \leq i, j \leq d}$, respectively. The vector $x$ and the matrix $A$ is nonnegative if $x_{i} \geq 0$ and $A_{i j} \geq 0,1 \leq i, j \leq d$, respectively. In this case, we write $x \geq 0$ and $A \geq O$. $\mathbb{R}^{d}$ can be endowed with any norms, but they are equivalent. A vector norm is denoted by $\|\cdot\|$ and the norm of a matrix in $\mathbb{R}^{d \times d}$ induced by this vector norm is also denoted by $\|\cdot\|$. The spectral radius of the matrix $A \in \mathbb{R}^{d \times d}$ is given by $\rho(A):=\lim _{n \rightarrow \infty}\left\|A^{n}\right\|^{1 / n}$, which is independent of the norm employed to calculate it.

A partial ordering is defined on $\mathbb{R}^{d}\left(\mathbb{R}^{d \times d}\right)$ by letting $x \leq y(A \leq B)$ if and only if $y-x \geq$ $0(B-A \geq O)$. The partial ordering enables us to define the sup, inf, lim sup, lim inf, and so forth for the sequences of vectors and matrices, which can also be determined componentwise and elementwise, respectively. It is known that $\rho(A) \leq \rho(|A|)$ for $A \in \mathbb{R}^{d \times d}$, and $\rho(A) \leq \rho(B)$ if $A, B \in \mathbb{R}^{d \times d}$ and $O \leq A \leq B$.

\section{The main results}

First, consider the nonconvolutional linear Volterra difference equation

$$
z(n+1)=\sum_{i=0}^{n} H(n, i) z(i)+h(n), \quad n \in \mathbb{Z}^{+},
$$


with initial condition

$$
z(0)=z_{0}
$$

Here, we assume

$\left(\mathrm{H}_{1}\right) z_{0} \in \mathbb{R}^{d}, H:=(H(n, i))_{0 \leq i \leq n}$ and $h:=(h(n))_{n \geq 0}$ are sequences with elements in $\mathbb{R}^{d \times d}$ and $\mathbb{R}^{d}$, respectively;

$\left(\mathrm{H}_{2}\right)$ for any fixed $i \geq 0$ the limit $H_{\infty}(i):=\lim _{n \rightarrow \infty} H(n, i)$ is finite and $\sum_{i=0}^{\infty}\left|H_{\infty}(i)\right|<\infty$;

$\left(\mathrm{H}_{3}\right)$ the matrix

$$
V:=\lim _{N \rightarrow \infty}\left(\lim _{n \rightarrow \infty} \sum_{j=N}^{n} H(n, j)\right)
$$

is finite;

$\left(\mathrm{H}_{4}\right)$ the matrix

$$
W:=\lim _{N \rightarrow \infty}\left(\limsup _{n \rightarrow \infty} \sum_{j=N}^{n}|H(n, j)|\right)
$$

is finite and $\rho(W)<1$;

$\left(\mathrm{H}_{5}\right)$ the limit $h(\infty):=\lim _{n \rightarrow \infty} h(n)$ is finite.

By a solution of (3.1), we mean a sequence $z:=(z(n))_{n \geq 0}$ in $\mathbb{R}^{d}$ satisfying (3.1) for any $n \in \mathbb{Z}^{+}$. It is clear that (3.1) with initial condition (3.2) has a unique solution.

Now, we are in a position to state our first main result.

Theorem 3.1. Assume $\left(H_{1}\right)-\left(H_{5}\right)$ are satisfied. Then for any $z_{0} \in \mathbb{R}^{d}$ the unique solution $z:=$ $(z(n))_{n \geq 0}$ of (3.1) and (3.2) has a finite limit at $\infty$ and it satisfies

$$
\lim _{n \rightarrow \infty} z(n)=(I-V)^{-1}\left(\sum_{i=0}^{\infty} H_{\infty}(i) z(i)+h(\infty)\right) .
$$

Under conditions $\left(\mathrm{H}_{3}\right)$ and $\left(\mathrm{H}_{4}\right)$

$$
|V|=\lim _{N \rightarrow \infty}\left(\lim _{n \rightarrow \infty}\left|\sum_{j=N}^{n} H(n, j)\right|\right) \leq W,
$$

and hence $\rho(W)<1$ yields $\rho(V)<1$, thus $I-V$ is invertible. On the other hand under our conditions the unique solution $z:=(z(n))_{n \geq 0}$ of (3.1) and (3.2) is a bounded sequence, therefore $\sum_{i=0}^{\infty} H_{\infty}(i) z(i)$ is finite, and (3.5) makes sense.

The second main result is dealing with the scalar Volterra difference equation

$$
\Delta x(n)=A x(n)+\sum_{j=-\infty}^{n} K(n-j) x(j)+g(n), \quad n \in \mathbb{Z}^{+},
$$


with the initial condition

$$
x(n)=\varphi(n), \quad n \in \mathbb{Z}^{-},
$$

where $A \in \mathbb{R}, K: \mathbb{Z}^{+} \rightarrow \mathbb{R}, g: \mathbb{Z}^{+} \rightarrow \mathbb{R}$, and $\varphi: \mathbb{Z}^{-} \rightarrow \mathbb{R}$ are given.

By a solution of the Volterra difference equation (3.7) we mean a sequence $x: \mathbb{Z} \rightarrow \mathbb{R}$ satisfies (3.7) for any $n \in \mathbb{Z}^{+}$.

In what follows, by $S$ we will denote the set of all initial sequence $\varphi: \mathbb{Z}^{-} \rightarrow \mathbb{R}$ such that for each $n \in \mathbb{Z}^{+}$

$$
\sum_{j=-\infty}^{-1} K(n-j) \varphi(j)
$$

exists.

It can be easily seen that for any initial sequence $\varphi \in S$, (3.7) has exactly one solution satisfying (3.8). This unique solution is denoted by $x(\varphi): \mathbb{Z} \rightarrow \mathbb{R}$ and it is called the solution of the initial value problem (3.7), (3.8).

The asymptotic representation of the solutions of (3.7) is given under the next condition.

(A) There exists a $\lambda_{0} \in \mathbb{R} \backslash\{0\}$ for which

$$
\begin{gathered}
\lambda_{0}=1+A+\sum_{i=0}^{\infty} K(i) \lambda_{0}^{-i} \\
\left|\lambda_{0}\right|^{-1} \sum_{i=1}^{\infty} i|K(i)|\left|\lambda_{0}\right|^{-i}<1 .
\end{gathered}
$$

From the theory of the infinite series, one can easily see that condition (A) yields

$$
r:=\limsup _{n \rightarrow \infty}|K(n)|^{1 / n}
$$

is finite. Moreover, the mapping $G:[r, \infty) \rightarrow[0, \infty]$, defined by

$$
G(\mu):= \begin{cases}\sum_{i=1}^{\infty} i|K(i)| \mu^{-(i+1)} & \text { if } \mu>r, \\ \sum_{i=1}^{\infty} i|K(i)| r^{-(i+1)} & \text { if } \mu=r>0, \\ \infty & \text { if } \mu=r=0,\end{cases}
$$

is real valued on $(r, \infty)$. It is also clear (see Section 5 ) that if there is an $n_{0} \geq 1$ such that $K\left(n_{0}\right) \neq 0$, and if $G(r) \geq 1$ then the equation

$$
G(\mu)=1
$$

has a unique solution, say $\mu_{1}$.

Now we formulate the following more explicit condition:

(B) either $K(n)=0, n \geq 1$, and

$$
1+A+K(0) \neq 0
$$


or there is an $n_{0} \geq 1$ with $K\left(n_{0}\right) \neq 0$, and

(i) $r$ defined in (3.12) is finite,

(ii) if $G(r) \geq 1$, then the constant $A$ satisfies either

$$
A>\mu_{1}-1-\sum_{i=0}^{\infty} K(i) \mu_{1}^{-i}
$$

or

$$
A<-\mu_{1}-1-\sum_{i=0}^{\infty}(-1)^{i} K(i) \mu_{1}^{-i}
$$

(iii) if $G(r)<1$, then the constant $A$ satisfies either

$$
A \geq r-1-\sum_{i=0}^{\infty} K(i) r^{-i}
$$

or

$$
A \leq-r-1-\sum_{i=0}^{\infty}(-1)^{i} K(i) r^{-i}
$$

Remark 3.2. Let $K: \mathbb{Z}^{+} \rightarrow \mathbb{R}$ be a sequence such that $K\left(n_{0}\right) \neq 0$ for some $n_{0} \geq 1$. It will be proved in Lemma 5.7 that there is at most one $\lambda_{0} \in \mathbb{C} \backslash\{0\}$ satisfying (3.10) and (3.11). It is an easy consequence of this statement that if $\lambda_{0} \in \mathbb{C} \backslash\{0\}$ satisfies (3.10) and (3.11), and $\lambda_{1} \in \mathbb{C} \backslash\left\{0, \lambda_{0}\right\}$ is a solution of (3.10), then $\left|\lambda_{1}\right|<\left|\lambda_{0}\right|$, thus $\lambda_{0}$ is the leading root of (3.10). Really, from the condition $\left|\lambda_{1}\right| \geq\left|\lambda_{0}\right|$ we have

$$
\sum_{i=1}^{\infty} i|K(i)|\left|\lambda_{1}\right|^{-(i+1)} \leq \sum_{i=1}^{\infty} i|K(i)|\left|\lambda_{0}\right|^{-(i+1)}<1,
$$

that is (3.11) holds for $\lambda_{1}$ instead of $\lambda_{0}$, and this contradicts the uniqueness of $\lambda_{0}$.

Now, we are ready to state our second result which will be proved in Section 5. This result shows that the implicit condition (A) and the explicit condition (B) are equivalent and the solutions of (3.7) can be asymptotically characterized by $\lambda_{0}^{n}$ as $n \rightarrow \infty$.

Theorem 3.3. Let $A \in \mathbb{R}, K: \mathbb{Z}^{+} \rightarrow \mathbb{R}, g: \mathbb{Z}^{+} \rightarrow \mathbb{R}$, and $\varphi \in S$ be given. Then

$(\alpha)$ Condition $(A)$ holds if and only if condition $(B)$ is satisfied.

$(\beta)$ If condition $(A)$ or equivalently condition $(B)$ holds, moreover

$$
\sum_{i=0}^{\infty} \lambda_{0}^{-i}\left(\sum_{j=-\infty}^{-1} K(i-j) \varphi(j)+g(i)\right)
$$

is finite, then for the solution $x(\varphi)$ of (3.7), (3.8) the limit $c[\varphi]:=\lim _{n \rightarrow \infty} \lambda_{0}^{-n} x(\varphi)(n)$ is finite and it obeys

$$
c[\varphi]=\left(\lambda_{0}-\sum_{i=1}^{\infty} i K(i) \lambda_{0}^{-i}\right)^{-1}\left(\sum_{i=0}^{\infty} \lambda_{0}^{-i}\left(\sum_{j=-\infty}^{-1} K(i-j) \varphi(j)+g(i)\right)+\lambda_{0} \varphi(0)\right) .
$$




\section{Examples and the discussion of the results}

In this section, we illustrate our results by examples and the interested reader could also find some discussions.

Example 4.1. Our Theorem 3.1 is given for system of equations, however the next example shows that this result is also new even in scalar case.

Let us consider the scalar nonconvolution Volterra difference equation

$$
z(n+1)=\sum_{j=1}^{n} \frac{(n-j)^{\alpha-1} j^{\beta-1}}{n^{\alpha+\beta-1}} z(j)+h(n), \quad n \geq 1,
$$

with the initial condition

$$
z(1)=z_{1},
$$

where $\left(a_{1}\right) \alpha, \beta>1$ and real, and $(h(n))_{n \geq 1}$ is a real sequence such that its limit $h(\infty):=$ $\lim _{n \rightarrow \infty} h(n)$ is finite.

Now, let the values $z_{0}, h(0)$ and the sequence $H:=(H(n, i))_{0 \leq i \leq n}$ be defined by

$$
\begin{aligned}
z_{0}: & =0, \quad h(0):=z_{1}, \\
H(n, i) & := \begin{cases}\frac{(n-i)^{\alpha-1} i^{\beta-1}}{n^{\alpha+\beta-1}}, & 1 \leq i \leq n, \\
0, & 0=i \leq n .\end{cases}
\end{aligned}
$$

Then, it can be easily seen that problem (4.1), (4.2) is equivalent to problem (3.1), (3.2).

We find that $H_{\infty}(i):=\lim _{n \rightarrow \infty} H(n, i)=0$ for any fixed $i \geq 0$.

It is known that

$$
\lim _{n \rightarrow \infty} \sum_{j=1}^{n} H(n, j)=\lim _{n \rightarrow \infty} \sum_{j=1}^{n} \frac{(n-j)^{\alpha-1} j^{\beta-1}}{n^{\alpha+\beta-1}}=B(\alpha, \beta),
$$

where $B(\alpha, \beta)$ is the well-known Beta function at $(\alpha, \beta)$ defined by

$$
B(\alpha, \beta):=\int_{0}^{1} t^{\alpha-1}(1-t)^{\beta-1} d t<1 .
$$

Using the nonnegativity of $H(n, i), 0 \leq i \leq n$, and Lemma 5.2 we have that

$$
O \leq V=W=\lim _{N \rightarrow \infty}\left(\lim _{n \rightarrow \infty} \sum_{j=N}^{n} H(n, j)\right)=\lim _{n \rightarrow \infty} \sum_{j=0}^{n} H(n, j)=B(\alpha, \beta)<1 .
$$

Now, one can easily see that for the sequences $h:=(h(n))_{n \geq 0}$ and $H:=(H(n, i))_{0 \leq i \leq n}$ all of the conditions of Theorem 3.1 are satisfied. Thus, by Theorem 3.1 we get that the solution $z:=(z(n))_{n \geq 1}$ of the initial value problem (4.1), (4.2) satisfies

$$
\lim _{n \rightarrow \infty} z(n)=\frac{h(\infty)}{1-B(\alpha, \beta)} .
$$

On the other hand, we know (see [23]) that

$$
\lim _{N \rightarrow \infty}\left(\lim _{n \rightarrow \infty} \sum_{j=N}^{n-N} H(n, j)\right)=\lim _{N \rightarrow \infty}\left(\lim _{n \rightarrow \infty} \sum_{j=N}^{n-N} \frac{(n-j)^{\alpha-1} j^{\beta-1}}{n^{\alpha+\beta-1}}\right)=B(\alpha, \beta) \neq 0,
$$

and hence in [20, Theorem 3.1] is not applicable. 
Example 4.2. Let $m \geq 1$ and $0<\tau_{1}<\cdots<\tau_{m}$ be given integers, and assume $K(i) \neq 0$ if $i \in$ $\left\{\tau_{1}, \ldots, \tau_{m}\right\}$ and $K(i)=0$ if $i \in \mathbb{Z}^{+} \backslash\left\{\tau_{1}, \ldots, \tau_{m}\right\}$. Then,

$$
\sum_{i=-\infty}^{n} K(n-i) x(i)=\sum_{i=0}^{\infty} K(i) x(n-i)=\sum_{k=1}^{m} K\left(\tau_{k}\right) x\left(n-\tau_{k}\right), \quad n \in \mathbb{Z}^{+} .
$$

Thus, (3.7) reduces to the delay difference equation

$$
\Delta x(n)=A x(n)+\sum_{k=1}^{m} K\left(\tau_{k}\right) x\left(n-\tau_{k}\right)+g(n), \quad n \in \mathbb{Z}^{+},
$$

and for any sequence $\varphi: \mathbb{Z}^{-} \rightarrow \mathbb{R}, \varphi \in S$ holds.

Since $K(n)=0$ for any large enough $n, r:=\lim _{n \rightarrow \infty}|K(n)|^{1 / n}=0$, moreover the function $G:[0, \infty) \rightarrow[0, \infty]$ defined in (3.13) satisfies

$$
G(\mu)= \begin{cases}\sum_{k=1}^{m} \tau_{k}\left|K\left(\tau_{k}\right)\right| \mu^{-\left(\tau_{k}+1\right),} & \text { if } \mu>0 \\ \infty, & \text { if } \mu=0\end{cases}
$$

Let $\mu_{1}>0$ be the unique value satisfying

$$
G\left(\mu_{1}\right)=\sum_{k=1}^{m} \tau_{k}\left|K\left(\tau_{k}\right)\right| \mu_{1}^{-\left(\tau_{k}+1\right)}=1
$$

Now, statement $(\alpha)$ of Theorem 3.3 is applicable and so the next statement is valid.

Proposition 4.3. For an $A \in \mathbb{R}$ there is a $\lambda_{0} \in \mathbb{R} \backslash\{0\}$ such that

$$
\begin{gathered}
\lambda_{0}=A+1+\sum_{k=1}^{m} K\left(\tau_{k}\right) \lambda_{0}^{-\tau_{k}}, \\
\left|\lambda_{0}\right|^{-1} \sum_{k=1}^{m} \tau_{k}\left|K\left(\tau_{k}\right)\right|\left|\lambda_{0}\right|^{-\tau_{k}}<1
\end{gathered}
$$

hold if and only if either

$$
A>\mu_{1}-1-\sum_{k=1}^{m} K\left(\tau_{k}\right) \mu_{1}^{-\tau_{k}}
$$

or

$$
A<-\mu_{1}-1-\sum_{k=1}^{m}(-1)^{\tau_{k}} K\left(\tau_{k}\right) \mu_{1}^{-\tau_{k}}
$$

is satisfied. 
Now, let $m=1, \tau_{1}:=l \in\{1, \ldots\}$ and $K(l) \neq 0$. Then $\mu_{1}=(l|K(l)|)^{1 /(l+1)}$, moreover $(4.14)$ and (4.15) reduce to

$$
\begin{gathered}
A>(l|K(l)|)^{1 /(l+1)}-1-K(l)(l|K(l)|)^{-l /(l+1)}, \\
A<-(l|K(l)|)^{1 /(l+1)}-1-(-1)^{l} K(l)(l|K(l)|)^{-l /(l+1)} .
\end{gathered}
$$

If especially $l=1$ and $K(1) \geq 0$, then $\mu_{1}=\sqrt{K(1)}$, moreover (4.14) and (4.15) are equivalent to the condition

$$
A \neq \sqrt{K(1)}-1-\sqrt{K(1)}=-\sqrt{K(1)}-1+\sqrt{K(1)}=-1
$$

Example 4.4. Let $q \in \mathbb{R} \backslash\{0\}$ and $K(i):=q^{i}, i \in \mathbb{Z}^{+}$. Then, (3.7) has the following form:

$$
\Delta x(n)=A x(n)+\sum_{j=-\infty}^{n} q^{n-j} x(j)+g(n), \quad n \in \mathbb{Z}^{+} .
$$

It is clear that $r:=\lim _{n \rightarrow \infty}\left|q^{n}\right|^{1 / n}=|q|$, and the function $G$ defined in (3.13) is given by

$$
G(\mu)= \begin{cases}\frac{|q|}{(\mu-|q|)^{2}}, & \text { if } \mu>|q|, \\ \infty, & \text { if } \mu=|q|,\end{cases}
$$

moreover $\mu_{1}=|q|+\sqrt{|q|}$ is the unique positive root of $G(\mu)=1$.

Thus statement $(\alpha)$ in Theorem 3.3 is applicable and as a corollary of it we obtain the following.

Proposition 4.5. There is a $\lambda_{0} \in \mathbb{R} \backslash\{0\}$ such that (3.10) and (3.11) hold with the sequence $K(i)=q^{i}$, $i \in \mathbb{Z}^{+}$, if and only if either

$$
A>|q|+\sqrt{|q|}-1-\frac{|q|+\sqrt{|q|}}{|q|+\sqrt{|q|}-q}
$$

or

$$
A<-(|q|+\sqrt{|q|})-1-\frac{|q|+\sqrt{|q|}}{|q|+\sqrt{|q|}+q} .
$$

Example 4.6. Let $c \in(1, \infty) \backslash \mathbb{Z}^{+}$and let $K(i):=\left(\begin{array}{c}c \\ i\end{array}\right), i \in \mathbb{Z}^{+}$. Here $\left(\begin{array}{c}c \\ i\end{array}\right)$ is the extended binomial coefficient, that is

$$
\left(\begin{array}{l}
c \\
i
\end{array}\right):=\frac{c(c-1) \cdots(c-(i-1))}{i !}, \quad i \in \mathbb{Z}^{+}
$$

In this case, $r=\lim _{n \rightarrow \infty}\left(\begin{array}{c}c \\ n\end{array}\right)^{1 / n}=1$ and by using the well-known properties of the binomial series, we find

$$
G(\mu)=\sum_{i=1}^{\infty} i\left(\begin{array}{l}
c \\
i
\end{array}\right) \mu^{-(i+1)}=\frac{1}{\mu^{2}} c\left(1+\frac{1}{\mu}\right)^{c-1}, \quad \text { if } \mu \geq 1 .
$$

Thus, $G(r)=G(1)=c 2^{c-1}>1$, therefore by statement $(\alpha)$ of Theorem 3.3 we get the following. 
Proposition 4.7. There is a $\lambda_{0} \in \mathbb{R} \backslash\{0\}$ such that (3.10) and (3.11) hold with the sequence $K(i)=$ $\left(\begin{array}{c}c \\ i\end{array}\right), i \in \mathbb{Z}^{+}$, if and only if either

$$
A>\mu_{1}-1-\left(1+\frac{1}{\mu_{1}}\right)^{c}
$$

or

$$
A<-\mu_{1}-1-\left(1-\frac{1}{\mu_{1}}\right)^{c}
$$

where $\mu_{1}$ is the unique positive solution of the equation

$$
\frac{1}{\mu^{2}} c\left(1+\frac{1}{\mu}\right)^{c-1}=1 \text {. }
$$

Example 4.8. Let $\alpha>3$ and $K(i):=1 / 2 i^{\alpha}, i \geq 1$, and $K(0):=0$. Then, (3.7) reduces to the special form

$$
\Delta x(n)=A x(n)+\sum_{j=-\infty}^{n-1} \frac{1}{2(n-j)^{\alpha}} x(j)+g(n), \quad n \in \mathbb{Z}^{+} .
$$

It is not difficult to see that $r=\left(1 / 2 n^{\alpha}\right)^{1 / n}=1$,

$$
G(\mu)=\sum_{i=1}^{\infty} \frac{1}{2 i^{\alpha-1}} \mu^{-(i+1)}, \quad \mu \geq 1
$$

and $G(1)<1$. From statement $(\alpha)$ of Theorem 3.3 we have the following.

Proposition 4.9. There is a $\lambda_{0} \in \mathbb{R} \backslash\{0\}$ such that (3.10) and (3.11) hold with the sequence $K(i)=$ $1 / 2 i^{\alpha}, i \geq 1$, if and only if either

$$
A>\sum_{i=1}^{\infty} \frac{1}{2 i^{\alpha}}=\frac{1}{2} \varsigma(\alpha)
$$

or

$$
A<-2-\sum_{i=1}^{\infty} \frac{(-1)^{i}}{2 i^{\alpha}}=-2-\left(\frac{1}{2^{\alpha-1}}-1\right) \sum_{i=1}^{\infty} \frac{1}{2 i^{\alpha}}=-2-\left(\frac{1}{2^{\alpha-1}}-1\right) \varsigma(\alpha)
$$

where $s$ is the well-known Riemann function.

\section{Proofs of the main theorems}

\subsection{Proof of Theorem 3.1}

To prove Theorem 3.1 we need the next result from [20]. 
I. Győri and L. Horváth

Theorem A. Let us consider the initial value problem (3.1), (3.2). Suppose that there are $M, N \in$ $\mathbb{Z}^{+}, M<N$ such that

$$
\begin{gathered}
\rho\left(\sup _{n \geq N} \sum_{i=M}^{n}|H(n, i)|\right)<1, \\
\sup _{n \geq M} \sum_{i=0}^{M}|H(n, i)|<\infty .
\end{gathered}
$$

Assume also that $\sup _{n \geq 0}|h(n)|<\infty$. Then, there is a nonnegative matrix $K \in \mathbb{R}^{d \times d}$, independent of $h$ and $z_{0}$, such that the solution $z$ of (3.1), (3.2) satisfies

$$
|z(n)| \leq K\left(\sup _{m \geq 0}|h(m)|+\left|z_{0}\right|\right), \quad n \geq 0 .
$$

Now, we prove some lemmas.

Lemma 5.1. The hypotheses of Theorem 3.1 imply that the hypotheses of Theorem A are satisfied, and hence the solution $z:=(z(n))_{n \geq 0}$ of (3.1), (3.2) is bounded.

Proof. Let $\epsilon>0$ be such that $\rho(W+\epsilon E)<1$. This can be satisfied because $\rho(W)<1$. Then, there is an $M_{0} \geq 0$ for which

$$
\limsup _{n \rightarrow \infty} \sum_{j=M_{0}}^{n}|H(n, j)|<W+\epsilon E,
$$

and hence for an $M \geq M_{0}$, we have

$$
\sum_{j=M_{0}}^{n}|H(n, j)|<W+\epsilon E, \quad n \geq M+1 .
$$

Thus,

$$
\sum_{j=M}^{n}|H(n, j)| \leq \sum_{j=M_{0}}^{n}|H(n, j)|<W+\epsilon E, \quad n \geq M+1
$$

therefore,

$$
\sup _{n \geq M+1} \sum_{j=M}^{n}|H(n, j)| \leq W+\epsilon E .
$$

But the matrices are nonnegative in the above inequality, thus

$$
\rho\left(\sup _{n \geq M+1} \sum_{j=M}^{n}|H(n, j)|\right) \leq \rho(W+\epsilon E)<1,
$$


and this shows (5.1). Since condition $\left(\mathrm{H}_{2}\right)$ holds, we get

$$
\lim _{n \rightarrow \infty} \sum_{j=0}^{M}|H(n, j)|=\sum_{j=0}^{M}\left|H_{\infty}(j)\right|
$$

therefore,

$$
\sup _{n \geq M} \sum_{j=0}^{M}|H(n, j)|<\infty
$$

thus (5.2) is satisfied.

In the next lemma we give an equivalent form of $\left(\mathrm{H}_{3}\right)$.

Lemma 5.2. Let $H:=(H(n, i))_{0 \leq i \leq n}$ be a sequence of real $d$ by $d$ matrices which satisfies $\left(H_{2}\right)$. Then, there exists a real $d$ by $d$ matrix $V$ such that

$$
\lim _{N \rightarrow \infty}\left(\limsup _{n \rightarrow \infty}\left|\sum_{j=N}^{n} H(n, j)-V\right|\right)=O
$$

if and only if

$$
\lim _{N \rightarrow \infty}\left(\lim _{n \rightarrow \infty} \sum_{j=N}^{n} H(n, j)\right)
$$

is finite. In both cases

$$
V=\lim _{N \rightarrow \infty}\left(\lim _{n \rightarrow \infty} \sum_{j=N}^{n} H(n, j)\right)=\lim _{n \rightarrow \infty} \sum_{j=0}^{n} H(n, j)-\sum_{j=0}^{\infty} H_{\infty}(j) .
$$

If $H$ satisfies $\left(H_{4}\right)$ too, and (5.11) holds, then $\rho(V)<1$.

Proof. First we show that

$$
\lim _{N \rightarrow \infty}\left(\lim _{n \rightarrow \infty} \sum_{j=N}^{n} H(n, j)\right)
$$

is finite if and only if

$$
\lim _{n \rightarrow \infty} \sum_{j=0}^{n} H(n, j)
$$

is finite, and in both cases

$$
\lim _{N \rightarrow \infty}\left(\lim _{n \rightarrow \infty} \sum_{j=N}^{n} H(n, j)\right)=\lim _{n \rightarrow \infty} \sum_{j=0}^{n} H(n, j)-\sum_{j=0}^{\infty} H_{\infty}(j) .
$$


I. Győri and L. Horváth

These come from $\left(\mathrm{H}_{2}\right)$, since

$$
\sum_{j=0}^{n} H(n, j)=\sum_{j=0}^{N} H(n, j)+\sum_{j=N+1}^{n} H(n, j), \quad n \geq N+1, N \geq 0 .
$$

Suppose $V$ is a real $d$ by $d$ matrix. Then, by $\left(\mathrm{H}_{2}\right)$ for every $N \geq 0$

$$
\begin{aligned}
& \underset{n \rightarrow \infty}{\limsup }\left|\sum_{j=N+1}^{n} H(n, j)-V\right| \\
& \quad=\max \left(\underset{n \rightarrow \infty}{\limsup } \sum_{j=0}^{n} H(n, j)-\sum_{j=0}^{N} H_{\infty}(j)-V,-\liminf _{n \rightarrow \infty} \sum_{j=0}^{n} H(n, j)+\sum_{j=0}^{N} H_{\infty}(j)+V\right),
\end{aligned}
$$

and hence

$$
\begin{aligned}
& \limsup _{N \rightarrow \infty}\left(\limsup _{n \rightarrow \infty}\left|\sum_{j=N+1}^{n} H(n, j)-V\right|\right) \\
& \quad=\max \left(\limsup _{n \rightarrow \infty} \sum_{j=0}^{n} H(n, j)-\sum_{j=0}^{\infty} H_{\infty}(j)-V,-\liminf _{n \rightarrow \infty} \sum_{j=0}^{n} H(n, j)+\sum_{j=0}^{\infty} H_{\infty}(j)+V\right) .
\end{aligned}
$$

Now, suppose that (5.11) holds. Then by (5.19), either

$$
\begin{gathered}
V=\limsup _{n \rightarrow \infty} \sum_{j=0}^{n} H(n, j)-\sum_{j=0}^{\infty} H_{\infty}(j), \\
-\liminf _{n \rightarrow \infty} \sum_{j=0}^{n} H(n, j)+\sum_{j=0}^{\infty} H_{\infty}(j)+V \leq O,
\end{gathered}
$$

or

$$
\begin{gathered}
V=\liminf _{n \rightarrow \infty} \sum_{j=0}^{n} H(n, j)-\sum_{j=0}^{\infty} H_{\infty}(j), \\
\limsup _{n \rightarrow \infty} \sum_{j=0}^{n} H(n, j)-\sum_{j=0}^{\infty} H_{\infty}(j)-V \leq O .
\end{gathered}
$$

Both of the previous cases implies that

$$
\limsup _{n \rightarrow \infty} \sum_{j=0}^{n} H(n, j) \leq \liminf _{n \rightarrow \infty} \sum_{j=0}^{n} H(n, j)
$$

which shows that

$$
\lim _{n \rightarrow \infty} \sum_{j=0}^{n} H(n, j)
$$


is finite and

$$
V=\lim _{n \rightarrow \infty} \sum_{j=0}^{n} H(n, j)-\sum_{j=0}^{\infty} H_{\infty}(j)
$$

As we have seen, this is equivalent with (5.12). If (5.12) is true or equivalently

$$
\lim _{n \rightarrow \infty} \sum_{j=0}^{n} H(n, j)
$$

is finite, then by (5.19)

$$
V:=\lim _{n \rightarrow \infty} \sum_{j=0}^{n} H(n, j)-\sum_{j=0}^{\infty} H_{\infty}(j)
$$

satisfies (5.11). $\rho(V)<1$ follows from $\left(\mathrm{H}_{4}\right)$. The proof is now complete.

Lemma 5.3. The hypotheses of Theorem 3.1 imply that

$$
c_{z}:=(I-V)^{-1}\left(\sum_{j=0}^{\infty} H_{\infty}(j) z(j)+h(\infty)\right)
$$

is the only vector satisfying the equation

$$
c_{z}=\sum_{j=0}^{\infty} H_{\infty}(j) z(j)+V c_{z}+h(\infty) .
$$

Proof. Since $\rho(V) \leq \rho(W)<1$ the matrix $I-V$ is invertible, which shows the uniqueness part of the lemma. On the other hand, by Lemma 5.1 we have that $z$ is a bounded sequence, and hence $\sum_{j=0}^{\infty} H_{\infty}(z) z(j)$ is finite. Thus, $c_{z}$ is well defined and satisfies (5.28). The proof is complete.

Lemma 5.4. The vector defined by (5.27) satisfies the relation

$$
c_{z}=\sum_{j=0}^{N} H(n, j) z(j)+\sum_{j=N+1}^{n} H(n, j) c_{z}+h(n)+g(n, N)
$$

for any $n>N \geq 0$, where the sequence $g(n, N) \in \mathbb{R}^{d}, n>N$, satisfies

$$
\lim _{N \rightarrow \infty}\left(\lim _{n \rightarrow \infty} g(n, N)\right)=0
$$

Proof. Let $n>N \geq 0$ be arbitrarily fixed and

$$
g(n, N):=\sum_{j=0}^{N}\left(H_{\infty}(j)-H(n, j)\right) z(j)+\sum_{j=N+1}^{\infty} H_{\infty}(j) z(j)+\left(V-\sum_{j=N+1}^{n} H(n, j)\right) c_{z}+h(\infty)-h(n) .
$$


But under the hypotheses of Theorem 3.1 we find

$$
\begin{aligned}
\limsup _{n \rightarrow \infty}|g(n, N)| \leq & \sum_{j=0}^{N} \lim _{n \rightarrow \infty}\left|H_{\infty}(j)-H(n, j)\right||z(j)|+\sum_{j=N+1}^{\infty}\left|H_{\infty}(j)\right||z(j)| \\
& +\limsup _{n \rightarrow \infty}\left|V-\sum_{j=N+1}^{n} H(n, j)\right|\left|c_{z}\right|+\lim _{n \rightarrow \infty}|h(\infty)-h(n)| \\
= & \sum_{j=N+1}^{\infty}\left|H_{\infty}(j)\right||z(j)|+\underset{n \rightarrow \infty}{\limsup }\left|V-\sum_{j=N+1}^{n} H(n, j)\right|\left|c_{z}\right|, \quad N \geq 0 .
\end{aligned}
$$

Now, by Lemma 5.2

$\limsup _{N \rightarrow \infty}\left(\limsup _{n \rightarrow \infty}|g(n, N)|\right) \leq \lim _{N \rightarrow \infty} \sum_{j=N+1}^{\infty}\left|H_{\infty}(j)\right||z(j)|+\lim _{N \rightarrow \infty}\left(\limsup _{n \rightarrow \infty}\left|V-\sum_{j=N+1}^{n} H(n, j)\right|\left|c_{z}\right|\right)=0$,

and hence (5.30) holds. On the other hand, it can be easily seen that by the above definition of $g(n, N)$ the relation (5.29) also holds. The proof is complete.

Now, we prove Theorem 3.1.

Proof. Let $n>N \geq 0$ be arbitrarily fixed. Then, (3.1) can be written in the form

$$
z(n+1)=\sum_{i=0}^{N} H(n, i) z(i)+\sum_{i=N+1}^{n} H(n, i) z(i)+h(n) .
$$

Subtracting (5.29) from the above equation, we get

$$
z(n+1)-c_{z}=\sum_{i=N+1}^{n} H(n, i)\left(z(i)-c_{z}\right)-g(n, N), \quad n>N \geq 0 .
$$

On the other hand, by Lemma $5.1, z:=(z(n))_{n \geq 0}$ is bounded and hence

$$
\eta:=\limsup _{n \rightarrow \infty}\left|z(n)-c_{z}\right|
$$

is finite. Let $\epsilon>0$ be arbitrarily fixed and $e:=(1, \ldots, 1)^{T}$. Then there is an $N_{0} \geq 0$ such that

$$
\left|z(n)-c_{z}\right| \leq \eta+\epsilon e, \quad n \geq N_{0}
$$

Thus, (5.35) yields

$$
\left|z(n+1)-c_{z}\right| \leq \sum_{i=N+1}^{n}|H(n, i)|(\eta+\epsilon e)+|g(n, N)|, \quad n>N \geq N_{0} .
$$

From this it follows:

$$
\eta=\limsup _{n \rightarrow \infty}\left|z(n)-c_{z}\right| \leq \limsup _{n \rightarrow \infty} \sum_{i=N+1}^{n}|H(n, i)|(\eta+\epsilon e)+\limsup _{n \rightarrow \infty}|g(n, N)|, \quad N \geq N_{0} .
$$


Thus,

$$
\eta \leq \lim _{N \rightarrow \infty}\left(\limsup _{n \rightarrow \infty} \sum_{i=N+1}^{n}|H(n, i)|\right)(\eta+\epsilon e)+\lim _{N \rightarrow \infty}\left(\limsup _{n \rightarrow \infty}|g(n, N)|\right)
$$

and hence Lemma 5.4 implies that

$$
\eta \leq W(\eta+\epsilon e)
$$

Since $W$ is a nonnegative matrix with $\rho(W)<1$, we have that $(I-W)^{-1} \geq O$. Thus,

$$
\eta \leq(I-W)^{-1} W \epsilon e \longrightarrow 0, \quad \epsilon \longrightarrow 0+
$$

and hence the proof of Theorem 3.1 is complete.

\subsection{Proof of Theorem 3.3}

Theorem 3.3 will be proved after some preparatory lemmas.

In the next lemma, we show that (3.7) can be transformed into an equation of the form (3.1) by using the transformation

$$
z(n)=\lambda_{0}^{-n} x(\varphi)(n), \quad n \in \mathbb{Z}^{+}
$$

Lemma 5.5. Under the conditions of Theorem 3.3, the sequence $z: \mathbb{Z}^{+} \rightarrow \mathbb{R}$ defined by (5.43) satisfies (3.1), where the sequences $H:\{(n, i) \mid 0 \leq i \leq n\} \rightarrow \mathbb{R}$ and $h: \mathbb{Z}^{+} \rightarrow \mathbb{R}$ are defined by

$$
\begin{gathered}
H(n, i):=-\lambda_{0}^{-1} \sum_{j=n-i+1}^{\infty} K(j) \lambda_{0}^{-j}, \quad 0 \leq i \leq n, \\
h(n):=\lambda_{0}^{-1} \sum_{i=0}^{n} \lambda_{0}^{-i}\left(\sum_{j=-\infty}^{-1} K(i-j) \varphi(j)+g(i)\right)+\varphi(0), \quad n \in \mathbb{Z}^{+} .
\end{gathered}
$$

Proof. Let $z:=(z(n))_{n \geq 0}$ be defined by (5.43). Then,

$$
x(\varphi)(n)=\lambda_{0}^{n} z(n), \quad n \in \mathbb{Z}^{+} .
$$

Thus,

$$
\Delta x(\varphi)(n)=\lambda_{0}^{n+1} z(n+1)-\lambda_{0}^{n} z(n)=\lambda_{0}^{n+1}(z(n+1)-z(n))+\left(\lambda_{0}^{n+1}-\lambda_{0}^{n}\right) z(n), \quad n \in \mathbb{Z}^{+} .
$$

On the other hand, from (3.7) it follows:

$$
\Delta x(\varphi)(n)=A x(\varphi)(n)+\sum_{j=0}^{n} K(n-j) x(\varphi)(j)+\sum_{j=-\infty}^{-1} K(n-j) \varphi(j)+g(n), \quad n \in \mathbb{Z}^{+} .
$$


Thus,

$$
\begin{aligned}
\Delta z(n)= & \lambda_{0}^{-(n+1)} \Delta x(\varphi)(n)+\left(\lambda_{0}^{-1}-1\right) z(n) \\
= & \lambda_{0}^{-(n+1)} A x(\varphi)(n)+\left(\lambda_{0}^{-1}-1\right) z(n)+\lambda_{0}^{-(n+1)} \sum_{j=0}^{n} K(n-j) x(\varphi)(j) \\
& +\lambda_{0}^{-(n+1)} \sum_{j=-\infty}^{-1} K(n-j) \varphi(j)+\lambda_{0}^{-(n+1)} g(n), \quad n \in \mathbb{Z}^{+},
\end{aligned}
$$

and hence

$$
\begin{aligned}
\Delta z(n)= & \left(\lambda_{0}^{-1} A+\lambda_{0}^{-1}-1\right) z(n)+\lambda_{0}^{-(n+1)} \sum_{j=0}^{n} K(n-j) \lambda_{0}^{j} z(j) \\
& +\lambda_{0}^{-(n+1)} \sum_{j=-\infty}^{-1} K(n-j) \varphi(j)+\lambda_{0}^{-(n+1)} g(n), \quad n \in \mathbb{Z}^{+} .
\end{aligned}
$$

But

$$
\sum_{i=0}^{n} \Delta z(i)=z(n+1)-z(0)=z(n+1)-\varphi(0), \quad n \in \mathbb{Z}^{+}
$$

Therefore,

$$
z(n+1)=\left(\lambda_{0}^{-1} A+\lambda_{0}^{-1}-1\right) \sum_{i=0}^{n} z(i)+\sum_{i=0}^{n} \sum_{j=0}^{i} \lambda_{0}^{-(i+1)} K(i-j) \lambda_{0}^{j} z(j)+h(n), \quad n \in \mathbb{Z}^{+}
$$

where $h: \mathbb{Z}^{+} \rightarrow \mathbb{R}$ is defined in (5.45). By interchanging the order of the summation we get

$$
\begin{aligned}
\sum_{i=0}^{n} \sum_{j=0}^{i} \lambda_{0}^{-(i+1)} K(i-j) \lambda_{0}^{j} z(j) & =\sum_{j=0}^{n} \sum_{i=j}^{n} \lambda_{0}^{-(i+1)} K(i-j) \lambda_{0}^{j} z(j)=\lambda_{0}^{-1} \sum_{j=0}^{n} \sum_{i=0}^{n-j} \lambda_{0}^{-i} K(i) z(j) \\
& =\lambda_{0}^{-1} \sum_{i=0}^{n} \sum_{j=0}^{n-i} \lambda_{0}^{-j} K(j) z(i), \quad n \in \mathbb{Z}^{+} .
\end{aligned}
$$

This and (5.52) yield

$$
z(n+1)=\sum_{i=0}^{n}\left(\lambda_{0}^{-1} A+\lambda_{0}^{-1}-1+\lambda_{0}^{-1} \sum_{j=0}^{n-i} \lambda_{0}^{-j} K(j)\right) z(i)+h(n), \quad n \in \mathbb{Z}^{+}
$$

By using the definition $\lambda_{0}$, we have

$$
\lambda_{0}^{-1}\left(A+1-\lambda_{0}+\sum_{j=0}^{n-i} \lambda_{0}^{-j} K(j)\right)=-\lambda_{0}^{-1} \sum_{j=n-i+1}^{\infty} \lambda_{0}^{-j} K(j), \quad 0 \leq i \leq n,
$$


and hence

$$
z(n+1)=\left(-\lambda_{0}^{-1} \sum_{i=0}^{n} \sum_{j=n-i+1}^{\infty} \lambda_{0}^{-j} K(j)\right) z(i)+h(n), \quad n \in \mathbb{Z}^{+} .
$$

But by using the definition of $H(n, i)$ in (5.44) the proof of the lemma is complete.

In the next lemma, we collect some properties of the function $G$ defined in (3.13).

Lemma 5.6. Let $K: \mathbb{Z}^{+} \rightarrow \mathbb{R}$ be a sequence such that $K\left(n_{0}\right) \neq 0$ for some $n_{0} \geq 1$ and

$$
r:=\limsup _{n \rightarrow \infty}|K(n)|^{1 / n}<\infty .
$$

Then, the function $G$ defined in (3.13) has the following properties.

(a) The series of functions

$$
\sum_{i=1}^{\infty} i|K(i)| \mu^{-(i+1)}, \quad \mu>0
$$

is convergent on $(r, \infty)$ and it is divergent on $(0, r)$.

(b) $\lim _{\mu \rightarrow \infty} G(\mu)=0$

(c) $\lim _{\mu \rightarrow r+} G(\mu)=G(r)$

(d) $G$ is strictly decreasing.

(e) If $G(r) \geq 1$, then the equation $G(\mu)=1$ has a unique solution.

Proof. (a) The root test can be applied. (b) The series of functions (5.58) is uniformly convergent on $[a, \infty)$ for every $a>r$, and this, together with $\lim _{\mu \rightarrow \infty} \mu^{-(i+1)}=0(i \geq 1)$, implies the result. (c) If $G(r)$ is finite, then the series of functions (5.58) is uniformly convergent on $[r, \infty)$, hence $G$ is continuous on $[r, \infty)$. Suppose now that $G(r)=\infty$ and $r>0$. Let $c>0$ be fixed and let $i_{0} \in \mathbb{Z}^{+}$such that

$$
\sum_{i=1}^{i_{0}} i|K(i)| r^{-(i+1)}>c
$$

Since

$$
\lim _{\mu \rightarrow r+} \sum_{i=1}^{i_{0}} i|K(i)| \mu^{-(i+1)}=\sum_{i=1}^{i_{0}} i|K(i)| r^{-(i+1)}>c,
$$

there is a $\delta>0$ such that

$$
G(\mu) \geq \sum_{i=1}^{i_{0}} i|K(i)| \mu^{-(i+1)}>c,
$$

whenever $\mu \in(r, r+\delta)$, and this shows $\lim _{\mu \rightarrow r+} G(\mu)=\infty$. Finally, we consider the case $r=$ 0 . Then, $\lim _{\mu \rightarrow r+} G(\mu)=\infty$ follows from the condition $K\left(n_{0}\right) \neq 0$. (d) The series of functions (5.58) can be differentiated term-by-term within $(r, \infty)$, and therefore $G^{\prime}(\mu)<0, \mu \in(r, \infty)$. Together with (c) this gives the claim. (e) We have only to apply (d), (c), and (b). The proof is complete. 
We are now in a position to prove Theorem 3.3.

Proof. (a) Let $K\left(n_{0}\right)=0$ for all $n \geq 1$. Then, it is easy to see that there is a $\lambda_{0} \in \mathbb{R} \backslash\{0\}$ such that (3.10) holds if and only if (3.15) is true, and in this case (3.11) is also satisfied. Suppose $K\left(n_{0}\right) \neq 0$ for some $n \geq 1$. Let $r$ be finite. By the root test, the series

$$
\sum_{i=0}^{\infty} K(i) \mu^{-i}, \quad \sum_{i=0}^{\infty}(-1)^{i} K(i) \mu^{-i}, \quad \mu>0
$$

are convergent for all $\mu>r$. Moreover, it can be easily verified that the series

$$
\sum_{i=0}^{\infty} K(i) r^{-i}, \quad \sum_{i=0}^{\infty}(-1)^{i} K(i) r^{-i}
$$

are absolutely convergent, whenever $G(r)$ is finite. Define the functions $F_{i}: D \rightarrow \mathbb{R}(i=1,2)$ by

$$
\begin{gathered}
F_{1}(\mu):=\mu-1-A-\sum_{i=0}^{\infty} K(i) \mu^{-i}, \\
F_{2}(\mu):=\mu+1+A+\sum_{i=0}^{\infty}(-1)^{i} K(i) \mu^{-i},
\end{gathered}
$$

where $D:=[r, \infty)$ if $G(r)$ is finite and $D:=(r, \infty)$, otherwise. The series of functions in (5.64) are uniformly convergent on $[a, \infty)$ for every $a \in D$, hence $F_{1}$ and $F_{2}$ are continuous. Further,

$$
\begin{aligned}
& F_{1}^{\prime}(\mu)=1+\sum_{i=1}^{\infty} i K(i) \mu^{-(i+1)} \geq 1-\sum_{i=1}^{\infty} i|K(i)| \mu^{-(i+1)}=1-G(\mu), \quad \mu>r \\
& F_{2}^{\prime}(\mu)=1-\sum_{i=1}^{\infty}(-1)^{i} i K(i) \mu^{-(i+1)} \geq 1-\sum_{i=1}^{\infty} i|K(i)| \mu^{-(i+1)}=1-G(\mu), \quad \mu>r .
\end{aligned}
$$

Let $\mu_{2}:=\mu_{1}$ if $G(r) \geq 1$, and let $\mu_{2}:=r$ if $G(r)<1$. It now follows from the previous inequalities and Lemma 5.6(d) that

$$
F_{i}^{\prime}(\mu)>0, \quad \mu>\mu_{2}, \quad i=1,2,
$$

and hence $F_{i}(i=1,2)$ is strictly increasing on $\left[\mu_{2}, \infty\right)$. It is immediate that $\lim _{\mu \rightarrow \infty} F_{i}(\mu)=\infty(i=$ $1,2)$. If (3.10) is hold for some $\lambda_{0} \in \mathbb{R} \backslash\{0\}$, then the convergence of the series $\sum_{i=0}^{\infty} K(i) \lambda_{0}^{-i}$ implies $r<\infty$. Suppose $r<\infty$. It is simple to see that there is a $\lambda_{0} \in \mathbb{R} \backslash\{0\}$ satisfying (3.10) if and only if either $F_{1}\left(\lambda_{0}\right)=0$ (in case $\lambda_{0}>0$ ) or $F_{2}\left(-\lambda_{0}\right)=0$ (in case $\lambda_{0}<0$ ). Moreover, the existence of a $\lambda_{0} \in \mathbb{R} \backslash\{0\}$ satisfying (3.11) is equivalent to either $\left|\lambda_{0}\right|>\mu_{1}$ (in case $G(r) \geq 1$ ) or $\left|\lambda_{0}\right| \geq r$ (in case $G(r)<1$ ). Now, the result follows from the properties of the functions $F_{i}(i=1,2)$. The proof of (a) is complete. (b) In virtue of Lemma 5.5 the proof of the theorem will be complete if we show that the sequences $H$ and $h$ satisfy the conditions $\left(\mathrm{H}_{2}\right)-\left(\mathrm{H}_{5}\right)$ in Section 3. Since the series $\sum_{i=0}^{\infty} K(i) \lambda_{0}^{-i}$ is convergent,

$$
H_{\infty}(i):=\lim _{n \rightarrow \infty} H(n, i)=\lim _{n \rightarrow \infty}\left(-\lambda_{0}^{-1} \sum_{j=n-i+1}^{\infty} \lambda_{0}^{-j} K(j)\right)=0, \quad i \in \mathbb{Z}^{+},
$$


and hence $\sum_{i=0}^{\infty}\left|H_{\infty}(i)\right|=0$. Thus $\left(\mathrm{H}_{2}\right)$ holds. Now, let $n \geq N \geq 0$ and consider $\sum_{i=N}^{n}|H(n, i)|$. In fact

$$
\begin{aligned}
\sum_{i=N}^{n}|H(n, i)| & =\sum_{i=N}^{n}\left|\lambda_{0}\right|^{-1}\left|\sum_{j=n-i+1}^{\infty} \lambda_{0}^{-j} K(j)\right| \\
& \leq \sum_{i=N}^{n}\left|\lambda_{0}\right|^{-1} \sum_{j=n-i+1}^{\infty}\left|\lambda_{0}\right|^{-j}|K(j)| \\
& =\sum_{l=1}^{n-N+1}\left|\lambda_{0}\right|^{-1} \sum_{j=l}^{\infty}\left|\lambda_{0}\right|^{-j}|K(j)| .
\end{aligned}
$$

Thus,

$$
\begin{aligned}
\limsup _{n \rightarrow \infty} \sum_{i=N}^{n}|H(n, i)| & \leq\left|\lambda_{0}\right|^{-1} \sum_{l=1}^{\infty} \sum_{j=l}^{\infty}\left|\lambda_{0}\right|^{-j}|K(j)| \\
& =\left|\lambda_{0}\right|^{-1} \sum_{j=0}^{\infty} \sum_{l=1}^{j}\left|\lambda_{0}\right|^{-j}|K(j)| \\
& =\left|\lambda_{0}\right|^{-1} \sum_{j=1}^{\infty} j\left|\lambda_{0}\right|^{-j}|K(j)|<1 .
\end{aligned}
$$

Thus,

$$
W:=\lim _{N \rightarrow \infty}\left(\limsup _{n \rightarrow \infty} \sum_{i=N}^{n}|H(n, i)|\right)
$$

is finite and $\rho(W)<1$. In a similar way, one can easily prove that

$$
V=\lambda_{0}^{-1} \lim _{N \rightarrow \infty}\left(\lim _{n \rightarrow \infty} \sum_{i=N}^{n} H(n, i)\right)=\lambda_{0}^{-1} \sum_{j=1}^{\infty} j \lambda_{0}^{-j} K(j)
$$

is also finite. It is also clear that

$$
h(\infty)=\lim _{n \rightarrow \infty} h(n)
$$

is finite. Thus, by Theorem 3.1 we get that the limit

$$
c[\varphi]=\lim _{n \rightarrow \infty} z(n)=\lim _{n \rightarrow \infty} \lambda_{0}^{-n} x(\varphi)(n)
$$

is finite and satisfies the required relation (3.22). The proof is now complete.

Lemma 5.7. Let $K: \mathbb{Z}^{+} \rightarrow \mathbb{R}$ be a sequence such that $K\left(n_{0}\right) \neq 0$ for some $n_{0} \geq 1$. Then, there is at most one $\lambda_{0} \in \mathbb{C} \backslash\{0\}$ satisfying (3.10) and (3.11). 
Proof. Suppose on the contrary that there exist two different numbers from $\mathbb{C} \backslash\{0\}$, denoted by $\lambda_{0}$ and $\lambda_{1}$, such that (3.10) and (3.11) hold. Then,

$$
\begin{gathered}
\lambda_{j}=1+A+\sum_{i=0}^{\infty} K(i)\left(\frac{1}{\lambda_{j}}\right)^{i}, \quad j=0,1, \\
\sum_{i=1}^{\infty} i|K(i)|\left|\lambda_{j}\right|^{-(i+1)}<1, \quad j=0,1 .
\end{gathered}
$$

It follows from (5.74), the mean value inequality and (5.75) that

$$
\begin{aligned}
\left|\lambda_{0}-\lambda_{1}\right| & \leq \sum_{i=1}^{\infty} i|K(i)|\left(\max \left(\frac{1}{\left|\lambda_{0}\right|}, \frac{1}{\left|\lambda_{1}\right|}\right)\right)^{i-1}\left|\frac{1}{\left|\lambda_{0}\right|}-\frac{1}{\left|\lambda_{1}\right|}\right| \\
& =\left(\max \left(\frac{1}{\left|\lambda_{0}\right|}, \frac{1}{\left|\lambda_{1}\right|}\right)\right)^{-2} \sum_{i=1}^{\infty} i|K(i)|\left(\max \left(\frac{1}{\left|\lambda_{0}\right|}, \frac{1}{\left|\lambda_{1}\right|}\right)\right)^{i+1}\left|\frac{1}{\left|\lambda_{0}\right|}-\frac{1}{\left|\lambda_{1}\right|}\right| \\
& <\left(\max \left(\frac{1}{\left|\lambda_{0}\right|}, \frac{1}{\left|\lambda_{1}\right|}\right)\right)^{-2}\left|\frac{1}{\left|\lambda_{0}\right|}-\frac{1}{\left|\lambda_{1}\right|}\right| \leq|| \lambda_{0}|-| \lambda_{1}||,
\end{aligned}
$$

and this is a contradiction.

\section{Acknowledgment}

This work was supported by Hungarian National Foundation for Scientific Research Grant no. K73274.

\section{References}

[1] R. P. Agarwal, Difference Equations and Inequalities: Theory, Methods, and Application, vol. 228 of Monographs and Textbooks in Pure and Applied Mathematics, Marcel Dekker, New York, NY, USA, 2nd edition, 2000.

[2] S. Elaydi, An Introduction to Difference Equations, Undergraduate Texts in Mathematics, Springer, New York, NY, USA, 3rd edition, 2005.

[3] W. G. Kelley and A. C. Peterson, Difference Equations: An Introduction with Application, Academic Press, Boston, Mass, USA, 1991.

[4] V. B. Kolmanovskii, E. Castellanos-Velasco, and J. A. Torres-Muñoz, "A survey: stability and boundedness of Volterra difference equations," Nonlinear Analysis: Theory, Methods \& Applications, vol. 53, no. 7-8, pp. 861-928, 2003.

[5] R. Medina, "Asymptotic behavior of Volterra difference equations," Computers \& Mathematics with Applications, vol. 41, no. 5-6, pp. 679-687, 2001.

[6] R. Medina and M. I. Gil, "The freezing method for abstract nonlinear difference equations," Journal of Mathematical Analysis and Applications, vol. 330, no. 1, pp. 195-206, 2007.

[7] Y. Song and C. T. H. Baker, "Admissibility for discrete Volterra equations," Journal of Difference Equations and Applications, vol. 12, no. 5, pp. 433-457, 2006.

[8] R. P. Agarwal and M. Pituk, "Asymptotic expansions for higher-order scalar difference equations," Advances in Difference Equations, vol. 2007, Article ID 67492, 12 pages, 2007.

[9] L. Berezansky and E. Braverman, "On exponential dichotomy, Bohl-Perron type theorems and stability of difference equations," Journal of Mathematical Analysis and Applications, vol. 304, no. 2, pp. 511-530, 2005. 
[10] S. Bodine and D. A. Lutz, "Asymptotic solutions and error estimates for linear systems of difference and differential equations," Journal of Mathematical Analysis and Applications, vol. 290, no. 1, pp. 343362, 2004.

[11] S. Bodine and D. A. Lutz, "On asymptotic equivalence of perturbed linear systems of differential and difference equations," Journal of Mathematical Analysis and Applications, vol. 326, no. 2, pp. 1174-1189, 2007.

[12] R. D. Driver, G. Ladas, and P. N. Vlahos, "Asymptotic behavior of a linear delay difference equation," Proceedings of the American Mathematical Society, vol. 115, no. 1, pp. 105-112, 1992.

[13] S. Elaydi, S. Murakami, and E. Kamiyama, "Asymptotic equivalence for difference equations with infinite delay," Journal of Difference Equations and Applications, vol. 5, no. 1, pp. 1-23, 1999.

[14] S. Elaydi, "Asymptotics for linear difference equations. II. Applications," in New Trends in Difference Equations, pp. 111-133, Taylor \& Francis, London, UK, 2002.

[15] J. R. Graef and C. Qian, "Asymptotic behavior of a forced difference equation," Journal of Mathematical Analysis and Applications, vol. 203, no. 2, pp. 388-400, 1996.

[16] I. Györi, "Sharp conditions for existence of nontrivial invariant cones of nonnegative initial values of difference equations," Applied Mathematics and Computation, vol. 36, no. 2, pp. 89-111, 1990.

[17] V. Kolmanovskii and L. Shaikhet, "Some conditions for boundedness of solutions of difference Volterra equations," Applied Mathematics Letters, vol. 16, no. 6, pp. 857-862, 2003.

[18] Z. H. Li, "The asymptotic estimates of solutions of difference equations," Journal of Mathematical Analysis and Applications, vol. 94, no. 1, pp. 181-192, 1983.

[19] W. F. Trench, "Asymptotic behavior of solutions of Poincaré recurrence systems," Computers $\mathcal{E}$ Mathematics with Applications, vol. 28, no. 1-3, pp. 317-324, 1994.

[20] J. A. D. Applelby, I. Győri, and D. W. Reynolds, "On exact convergence rates for solutions of linear systems of Volterra difference equations," Journal of Difference Equations and Applications, vol. 12, no. 12, pp. 1257-1275, 2006.

[21] Ch. G. Philos and I. K. Purnaras, “The behavior of solutions of linear Volterra difference equations with infinite delay," Computers E Mathematics with Applications, vol. 47, no. 10-11, pp. 1555-1563, 2004.

[22] Ch. G. Philos and I. K. Purnaras, "On linear Volterra difference equations with infinite delay," Advances in Difference Equations, vol. 2006, Article ID 78470, 28 pages, 2006.

[23] I. Győri and L. Horváth, "Limit theorems for discrete sumsand convolutions," submitted. 